\title{
Joint Wireless Positioning and Emitter Identification in DVB-T Single Frequency Networks
}

\author{
Liang Chen, Lie-Liang Yang, Jun Yan and Ruizhi Chen
}

\begin{abstract}
Digital television (DTV) signal has been recognized as a promising signal for navigation and positioning. However, due to the single frequency network (SFN) transmission within the European standard digital video broadcasting terrestrial (DVB-T) system, emitter confusion problem occurs in navigation and positioning, resulting in that a receiver is unable to know from which emitter a received signal comes. In this paper, we consider the wireless positioning with emitter confusion problem in DVB-T SFN networks. A joint wireless positioning and emitter identification algorithm is proposed, which is based on the expectation maximization (EM) method. The proposed algorithm is tested in a scenario, where signals are received from 3 to 5 emitters. Simulation results show that, relying on more than 3 emitters used in the tests for $2 D$ positioning, the EM assisted positioning algorithm is feasible to achieve accurate positioning results in the existence of the emitter confusion problem. Our studies show that the performance achieved by the proposed algorithm approaches the Cramér-Rao bound (CRB). Furthermore, the proposed algorithm is effective to identify the DTV emitters, and the positioning performance is robust to the emitter identification error. Additionally, our methodology is general, and can be employed for time of arrival (TOA) based positioning in any SFNs.
\end{abstract}

Index Terms-DVB-T, single frequency network (SFN), expectation maximization (EM), Cramér-Rao Lower bound (CRLB), wireless positioning

\section{INTRODUCTION}

Terrestrial television signals have been designed for both indoor and outdoor reception. Recently, wireless positioning using digital television (DTV) signals has attracted a growing interest after the DTV systems have been put into operation for massive users. It has been recognized that the DTV signals have a range of advantages for positioning, when compared to the Global Navigation Satellite Systems (GNSS) [1]. Based on the DTV signals, wireless positioning benefits from a higher transmission power [2], larger signal transmission bandwidth [3], less Doppler effects and ionosphere disturbance [4], and lower carrier frequency, hence resulting in better diffraction performance and better receiving quality for urban and

L. Chen is with State Key Laboratory of Information Engineering in Surveying, Mapping and Remote Sensing, Wuhan University, Wuhan 430072, China and also with Collaborative Innovation Center of Geospatial Technology, Wuhan 430079, China (e-mail: 1.chen@whu.edu.cn). L.-L. Yang is with the faculty of Electronics and Computer Science, University of Southampton, University Road, Southampton, SO17 1BJ, United Kingdom, email: 1ly@ecs.soton.ac.uk. J. Yan is with Nanjing University of Posts and Telecommunications, Nanjing 210003, China, email: yanj@njupt.edu.cn. R Chen is with the State Key Laboratory of Information Engineering in Surveying, Mapping and Remote Sensing, Wuhan University, Wuhan 430072, China and also with Collaborative Innovation Center of Geospatial Technology, Wuhan 430079, China (e-mail: ruizhi.chen@whu.edu.cn)

Manuscript received December 14, 2016; revised April 3, 2017; accepted April 12, 2017.; (Corresponding author: Liang Chen.) indoor propagation [5]. Furthermore, it is cost effective, due to the reuse of existing DTV facilities [6]. The principles and recent progress in research about the DTV signal assisted navigation and positioning have been overviewed in detail in [7], [8].

Among all the terrestrial DTV standards, the European standard DVB-T [3] has been employed in most countries worldwide. In the DVB-T based systems, multiple emitters are deployed to cover a region by transmitting the same signals on the same frequency band, which is hence called as the single frequency network (SFN) [3]. The SFN is efficient in terms of the use of frequency resource. However, in positioning, SFN generates the emitter confusion problem that a positioning receiver is unable to know which DTV stations it receives signals from.

The emitter confusion problem has been addressed in some literature [9], [10], [11], [12]. Specifically, the watermarking techniques have been proposed for the emitter identification in DTV systems, which include the systems based on the Advanced Television Systems Committee (ATSC) standards [9] and that on the DVB-T2 standards [10]. However, applying the watermarking techniques has to change the structure of the transmitted DTV signals, which leads to extra cost for updating the whole DTV system. By contrast, the authors of [11] have proposed a reverse positioning technique, while the authors in [12] have proposed to use mobile velocity information, in order to avoid the emitter confusion problem. However, these techniques are only suitable for the positioning receivers in moving, but not suitable for the static positioning receivers.

In this paper, we propose a novel approach, namely the expectation maximization assisted positioning (EMP) algorithm, which jointly estimates a user's position and identifies the DTV emitters. Our EMP algorithm iteratively identifies the DTV emitters and estimates the position of the receiver, with the aid of the pseudo-ranges measured by the receiver based on its received signals from the different DTV stations. In comparison with the existing approaches [9], [10], our proposed method does not require to modify the structure of the existing DTV signals. Hence, it is suitable for both static and mobile receivers to carry out their positioning. Additionally, we should note that, although our method is derived and analyzed in the context of the DVB-T systems, the methodology is in fact very general, which may be employed in any SFN based systems for the time of arrrival (TOA) based positioning.

The rest of the paper is organized as follows. Section II presents the system model and formulates the problem of DTV-based positioning without knowledge about emitter la- 
bels. In Section III, we detail the solution to the problem. Section IV shows the testing results and provides corresponding discussion. Finally, in Section V, the conclusions from studies are summarized.

\section{SYSTEM MODEL AND PROBLEM FORMULATION}

\section{A. TOA Estimation with DVB-T signals}

The DVB-T standard uses the OFDM (orthogonal frequency division multiplexing) modulation to achieve robust transmission in multipath scenarios. In DVB-T systems, OFDM signals are specified by three parameters, namely, the number of subcarriers or the FFT (fast Fourier transform) size, the length of cyclic prefix (CP) and the sampling period. The options (or modes) for setting up these parameters are provided in the ETSI DVB-T standard [3]. In DVB-T systems, signals are continuously transmitted and every OFDM symbol is transmitted within a fixed duration of time. The pilot subcarriers in each OFDM symbol are given by a known Pseudo-Random Binary Sequence (PRBS) with boosted power. From the perspective of wireless positioning, the good autocorrelation property of the PRBS with boosted power and the continuous transmission are helpful to accurately acquire and track the signals for TOA estimation. Moreover, in DVB-T systems, multiple emitters are suggested to be coordinated to the GPS time and simultaneously transmit the same DVB-T signals in the same frequency band, forming the so-called SFN transmission. Therefore, multiple emitters supported by network synchronization make the timing-based estimation required for wireless positioning available.

In our previous work [7], a software defined DVB-T receiver has been developed to estimate the TOA of DVB-T signals for positioning. The results obtained from the field test campaign show that the TOA tracking on real DVB-T signals is capable of achieving good ranging accuracy, with the estimation error within 1 - 4 meters depending on the practical signal to noise ratio $(\mathrm{SNR})$.

\section{B. System Model}

We consider a user that can simultaneously receive signals from $M$ DVB-T stations located within an area covered by a DVB-T SFN network. The east-north (X-Y) coordinates of the user location is expressed as $\boldsymbol{x}=\left[\begin{array}{ll}x_{r} & y_{r}\end{array}\right]^{T}$, and the coordinates of the $i$ th DVB-T station is expressed as $c_{i}=\left[\begin{array}{ll}x_{i} & y_{i}\end{array}\right]^{T}$, $\{i \in 1,2, \ldots, M\}$. In DVB-T networks, the distance between a DVB-T station and a user can be measured by the TOA estimation [7]. Let $d_{i}$ denote the distance between the user at $\boldsymbol{x}$ and the $i$ th DVB-T station at $\boldsymbol{c}_{i}$, which can be expressed as

$$
d_{i} \triangleq h_{i}(\boldsymbol{x})=\left\|\boldsymbol{c}_{i}-\boldsymbol{x}\right\|, i=1,2, \ldots, M
$$

For the sake of simplicity, let us consider only the line-of-sight (LOS) transmission environments. In this case, the distance measurement is only corrupted by the system measurement noise expressed as $n_{i}$ for the $i$ th DVB-T station, which can be modeled as an independent and identically distributed (i.i.d.) white Gaussian noise $\mathrm{N}\left(0, \sigma_{i}^{2}\right)$ with zero mean and a variance of $\sigma_{i}^{2}$. Consequently, when $M$ DVB-T stations are considered,

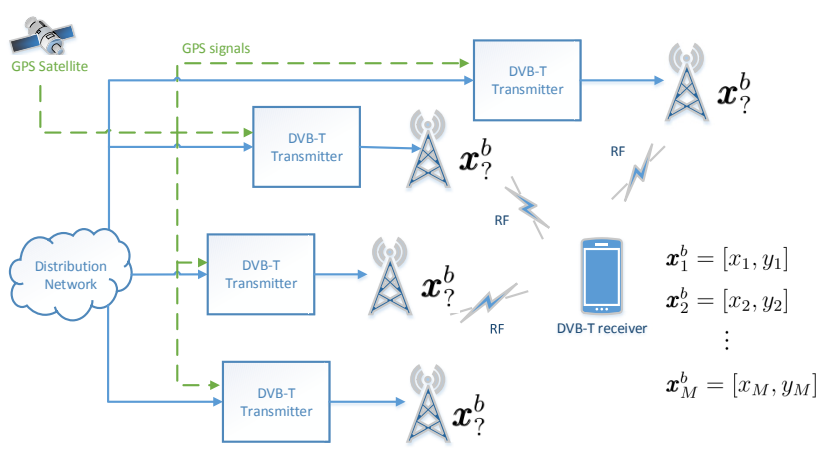

Fig. 1. Illustation of positioning confusion in DVB-T SFN.

the vector collecting the measurements of the distances from the $M$ DVB-T stations to $x$ can be written as

$$
z=h(x)+n
$$

where $z=\left[z_{1}, z_{2}, \cdots, z_{M}\right]^{T}, \quad \boldsymbol{h}(\boldsymbol{x})=$ $\left[h_{1}(\boldsymbol{x}), h_{2}(\boldsymbol{x}), \cdots, h_{M}(\boldsymbol{x})\right]^{T}$ and $\boldsymbol{n}=\left[n_{1}, n_{2}, \cdots, n_{M}\right]^{T}$. As $n_{i} \sim \mathrm{N}\left(0, \sigma_{i}^{2}\right)$, we have $\boldsymbol{n} \sim \mathcal{N}(\mathbf{0}, \boldsymbol{\Sigma})$, where $\boldsymbol{\Sigma}=\operatorname{diag}\left\{\sigma_{1}^{2}, \sigma_{2}^{2}, \cdots, \sigma_{M}^{2}\right\}$ is a diagonal matrix.

In DVB-T networks, the DVB-T stations' coordinates are fixed and can be assumed to be known to the positioning receiver by saving them in its memory. However, due to the property of the SFN, the label of a DVB-T station is not necessarily transmitted. Consequently, even though a positioning receiver knows the coordinates of the DVB-T stations invoked, it may be unable to identify from which DVB-T stations the signals are received, generating the emitter confusion problem. For example, in Fig. 1, when given the $M$ distsnces from the $M$ DVB-T stations to a positioning receiver, there may possibly exist $M$ ! DVB-T distributions yielding these distances, when we consider all the possible combinations of the distances from the DVB-T stations. Therefore, one of the important tasks in DVB-T based positioning is to identify the DVB-T stations, in order for a receiver to know from which DVB-T stations it receives signals and, ultimately, to calculate accurately its own location. Based on the above discussion, our positioning problem is formulated as follows.

\section{Problem Formulation}

Assume that the receiver knows a set of $M$ surrounding emitters expressed as $\{\mathbf{T x}\}$, from which the signals are received. Define the set of permutations generated by $\{\mathbf{T x}\}$ as $\mathbf{S} \triangleq\left\{\boldsymbol{s}_{1}, \cdots, \boldsymbol{s}_{L}\right\}$, where $L$ is equal to the factorial of the total $M$ emitters in $\{\mathbf{T x}\}$, i.e., $L=M$ !. For a permutation $\boldsymbol{s}_{j}=\left[s_{j}^{1}, s_{j}^{2}, \cdots, s_{j}^{M}\right], j \in\{1, \cdots, L\}$, a number $i \in\{1, \cdots, M\}$ is transformed to a number $s_{j}^{i}$, i.e. $\boldsymbol{s}_{j}(i)=s_{j}^{i}$, where $s_{j}^{i} \in\{1, \cdots, M\}$. Accordingly, the pseudo range measurement $z_{i}$ between the imaginary DVB-T station at $\boldsymbol{c}_{s_{j}^{i}}$ and the receiver is

$$
z_{i}=\left\|\boldsymbol{c}_{s_{j}^{i}}-\boldsymbol{x}\right\|+n_{i}
$$

As we know, within the set $s$, there is only one permutation, say $\boldsymbol{s}_{j}$, which corresponds to the actually received pseudo- 
ranges $z$. Thus, the positioning problem in the DVB-T SFN consists of the sub-problem of finding the right permutation of $\boldsymbol{s}_{j}$, and the sub-problem of estimating $\boldsymbol{x}$ based on the pseudo range measurements $z$.

In this paper, we propose a method, which jointly identifies the DTV emitters and estimates the receiver's location by maximizing the likelihood of the observed range measurements. Furthermore, an EMP algorithm is designed in order to find the solution, as formulated as follows.

\section{EXPECTATION MAXIMIZATION ASSISTED POSITIONING}

Based on the observed pseudo-range vector $z$, as shown in (2), the maximum likelihood estimation (MLE) can be applied, which estimates the receiver's position $x$ according to the optimization of

$$
\hat{\boldsymbol{x}}=\arg \max _{\boldsymbol{x}} p(\boldsymbol{z} \mid \boldsymbol{x})
$$

where

$$
p(\boldsymbol{z} \mid \boldsymbol{x})=\frac{1}{(2 \pi)^{M / 2}|\boldsymbol{\Sigma}|^{1 / 2}} e^{-\frac{1}{2}(\boldsymbol{z}-\boldsymbol{h}(\boldsymbol{x}))^{T} \boldsymbol{\Sigma}^{-1}(\boldsymbol{z}-\boldsymbol{h}(\boldsymbol{x}))}
$$

Taking the logarithm on both sides of (5) and neglecting the additive constants, it is explicit that the maximization of (5) is equivalent to the optimization of

$$
\hat{\boldsymbol{x}}=\arg \min _{\boldsymbol{x}}\left\{(\boldsymbol{z}-\boldsymbol{h}(\boldsymbol{x}))^{T} \boldsymbol{\Sigma}^{-1}(\boldsymbol{z}-\boldsymbol{h}(\boldsymbol{x})\}\right.
$$

However, in our problem, the labels of the DVB-T emitters are unknown, making the receiver unable to distinguish the signals received from the different emitters. In this case, even the receiver has the knowledge of the distances from the $M$ surrounding DVB-T emitters, it is still unable to calculate its position directly, when considering that there are in total $L$ possible permutations for a set of the measurements $\boldsymbol{z}$.

In order to solve the problem, we introduce the permutation variable $s$ as the missing data in the MLE. Then, the logarithm of $p(\boldsymbol{z} \mid \boldsymbol{x})$ can be rewritten as [13] ,

$$
\log p(\boldsymbol{z} \mid \boldsymbol{x})=\log p(\boldsymbol{z}, \boldsymbol{s} \mid \boldsymbol{x})-\log p(\boldsymbol{s} \mid \boldsymbol{z}, \boldsymbol{x})
$$

Upon taking the expectations on both sides with respect to the distribution $\log p\left(\boldsymbol{s} \mid \boldsymbol{z}, \boldsymbol{x}^{\text {old }}\right)$, where $\boldsymbol{x}^{\text {old }}$ is the current estimation of the position, we obtain

$$
\begin{aligned}
\log p(\boldsymbol{z} \mid \boldsymbol{x}) & =\sum_{\boldsymbol{s}} p\left(\boldsymbol{s} \mid \boldsymbol{z}, \boldsymbol{x}^{\text {old }}\right) \log p(\boldsymbol{z}, \boldsymbol{s} \mid \boldsymbol{x})-\sum_{\boldsymbol{s}} p\left(\boldsymbol{s} \mid \boldsymbol{z}, \boldsymbol{x}^{\mathrm{old}}\right) \log p(\boldsymbol{s} \mid \\
& =\mathbf{E}_{\text {old }}(\log p(\boldsymbol{z}, \boldsymbol{s} \mid \boldsymbol{x}))-\mathbf{E}_{\text {old }}(\log p(\boldsymbol{s} \mid \boldsymbol{z}, \boldsymbol{x}))
\end{aligned}
$$

where $\mathbf{E}_{\text {old }}$ is the expectation of $\boldsymbol{s}$ with respect to the distribution $p\left(\boldsymbol{s} \mid \boldsymbol{z}, \boldsymbol{x}^{\text {old }}\right)$. Note that, in (8) the left-hand side is the same as that in (7), as it does not depend on $s$. According to the Gibbs' inequality, the last term of $\mathbf{E}_{\text {old }}(\log p(\boldsymbol{s} \mid \boldsymbol{z}, \boldsymbol{x}))$ on the right side of (8) is maximized at $x=x^{\text {old }}$. Therefore, if we choose $\boldsymbol{x}$ to improve $\mathbf{E}_{\mathrm{old}}(\log p(\boldsymbol{z}, \boldsymbol{s} \mid \boldsymbol{x}))$ beyond $\mathbf{E}_{\text {old }}\left(\log p\left(\boldsymbol{z}, \boldsymbol{s} \mid \boldsymbol{x}^{\text {old }}\right)\right), \log p(\boldsymbol{z} \mid \boldsymbol{x})$ will be similarly improved beyond $\log p\left(\boldsymbol{z} \mid \boldsymbol{x}^{\text {old }}\right)$. In other words, the term of
$\mathbf{E}_{\text {old }}(\log p(\boldsymbol{z}, \boldsymbol{s} \mid \boldsymbol{x}))$, viewed as the expected complete-data loglikelihood [13], can be used as the cost function for maximization of $\log p(\boldsymbol{z} \mid \boldsymbol{x})$, which for simplicity can be denoted as

$$
\boldsymbol{Q}\left(\boldsymbol{x}, \boldsymbol{x}^{\mathrm{old}}\right)=\sum_{\boldsymbol{s}} p\left(\boldsymbol{s} \mid \boldsymbol{z}, \boldsymbol{x}^{\text {old }}\right) \log p(\boldsymbol{z}, \boldsymbol{s} \mid \boldsymbol{x})
$$

According to [14], with the aid of the missing data $s$, the EM algorithm is capable of converging to a local maximum of the log-likelihood function $\boldsymbol{Q}\left(\boldsymbol{x}, \boldsymbol{x}^{\text {old }}\right)$.

Let us factorize the complete-data likelihood as

$$
p(\boldsymbol{z}, \boldsymbol{s} \mid \boldsymbol{x})=p(\boldsymbol{z} \mid \boldsymbol{s}, \boldsymbol{x}) p(\boldsymbol{s} \mid \boldsymbol{x})
$$

where $p(s \mid x)$ acts as the a-priori knowledge about the true positions of the DVB-T stations. However, due to the lack of this knowledge, we assume the uniform a-priori of $s$. Meanwhile, we can assume that the selection of the possible permutation $\boldsymbol{s}$ is independent of $\boldsymbol{x}$, i.e. $p(\boldsymbol{s} \mid \boldsymbol{x})=p(\boldsymbol{s})$. Then, $\boldsymbol{Q}\left(\boldsymbol{x}, \boldsymbol{x}^{\text {old }}\right)$ in (9) can be further factorized as

$$
\begin{aligned}
\boldsymbol{Q}\left(\boldsymbol{x}, \boldsymbol{x}^{\text {old }}\right) & =\sum_{\boldsymbol{s}} p\left(\boldsymbol{s} \mid \boldsymbol{z}, \boldsymbol{x}^{\text {old }}\right) \log p(\boldsymbol{z} \mid \boldsymbol{s}, \boldsymbol{x})+\sum_{\boldsymbol{s}} p\left(\boldsymbol{s} \mid \boldsymbol{z}, \boldsymbol{x}^{\text {old }}\right) \log p(\boldsymbol{s}) \\
& =\boldsymbol{Q}_{1}\left(\boldsymbol{x}, \boldsymbol{x}^{\text {old }}\right)+\boldsymbol{Q}_{2}
\end{aligned}
$$

where by definition, $\quad \boldsymbol{Q}_{1}\left(\boldsymbol{x}, \boldsymbol{x}^{\text {old }}\right)$ = $\sum_{\boldsymbol{s}} p\left(\boldsymbol{s} \mid \boldsymbol{z}, \boldsymbol{x}^{\text {old }}\right) \log p(\boldsymbol{z} \mid \boldsymbol{s}, \boldsymbol{x}), \quad$ and $\quad \boldsymbol{Q}_{2}=$ $\sum_{\boldsymbol{s}} p\left(\boldsymbol{s} \mid \boldsymbol{z}, \boldsymbol{x}^{\text {old }}\right) \log p(\boldsymbol{s})$. Explicitly, $\boldsymbol{Q}_{2}$ is a constant, as it is not depended on $x$. Consequently, the maximization of $\mathrm{Q}\left(x, x^{\text {old }}\right)$ with respect to $x$ is equivalent to maximizing $Q_{1}\left(x, x^{\text {old }}\right)$.

\section{A. Derivation of Algorithm}

The EMP algorithm is divided into an E-step and an M-step, which are detailed below.

1) E-step: Given the current estimate of the position $x^{\text {old }}$, the conditional distribution of $p\left(\boldsymbol{s} \mid \boldsymbol{z}, \boldsymbol{x}^{\text {old }}\right)$ can be obtained by Bayes theorem as:

$$
p\left(\boldsymbol{s} \mid \boldsymbol{z}, \boldsymbol{x}^{\text {old }}\right) \propto p\left(\boldsymbol{z} \mid \boldsymbol{s}, \boldsymbol{x}^{\text {old }}\right) p\left(\boldsymbol{s} \mid \boldsymbol{x}^{\text {old }}\right)
$$

In (12), when we assume the uniform distribution of $s$, then we have $p\left(\boldsymbol{s} \mid \boldsymbol{x}^{\text {old }}\right)=p(\boldsymbol{s})$. According to the measurement $z$ mo)del of (2), when given a permutation $s, p\left(z \mid s, x^{\text {old }}\right)$ obeys a Gaussian distribution with the mean of $\boldsymbol{h}_{\boldsymbol{s}}\left(\boldsymbol{x}^{\text {old }}\right)$ and covariance matrix $\boldsymbol{\Sigma}$. Furthermore, let us define $\gamma_{\boldsymbol{s}}^{\text {old }}=p\left(\boldsymbol{s} \mid \boldsymbol{z}, \boldsymbol{x}^{\text {old }}\right)$, which represents the weight coefficient of the permutation of $s$ obtained from the current estimate $x^{\text {old }}$ and the measurements $z$. Thus, for the E-step, we have

$$
\boldsymbol{Q}_{1}\left(\boldsymbol{x}, \boldsymbol{x}^{\mathrm{old}}\right)=\sum_{\boldsymbol{s}} \gamma_{\boldsymbol{s}}^{\text {old }} \log p(\boldsymbol{z} \mid \boldsymbol{s}, \boldsymbol{x})
$$

where it can be shown that

$$
\gamma_{\boldsymbol{s}}^{\text {old }}=\frac{1}{L} \mathcal{N}\left(\boldsymbol{x}^{\text {old }} ; \boldsymbol{h}_{\boldsymbol{s}}\left(\boldsymbol{x}^{\mathrm{old}}\right), \Sigma\right)
$$


2) M-step: Given the weight coefficient $\gamma_{\boldsymbol{s}}^{\text {old }}$ derived from the E-step, the maximization step (M-step) is to find $\boldsymbol{x}$, so that

$$
\boldsymbol{x}=\arg \max _{\boldsymbol{x}} \mathbf{Q}_{1}\left(\boldsymbol{x}, \boldsymbol{x}^{\text {old }}\right)
$$

Upon substituting (5) into (13) and then into (15), taking the logarithms as well as neglecting the additive constants, it is explicit that the maximization of (15) is equivalent to the optimization problem of

$$
\hat{\boldsymbol{x}}=\arg \min _{\boldsymbol{x}}\left\{\sum_{\boldsymbol{s}}\left(\boldsymbol{z}-\boldsymbol{h}_{\boldsymbol{s}}(\boldsymbol{x})\right)^{T}\left(\Sigma / \gamma_{\boldsymbol{s}}^{\text {old })}\right)^{-1}\left(\boldsymbol{z}-\boldsymbol{h}_{\boldsymbol{s}}(\boldsymbol{x})\right)\right\}
$$

Let us define $e_{s}(x)=z-h_{s}(x), e(x)=$ $\left[\boldsymbol{e}_{\boldsymbol{s}_{1}}^{T}(\boldsymbol{x}), \cdots, \boldsymbol{e}_{\boldsymbol{s}_{L}}^{T}(\boldsymbol{x})\right]^{T}, \quad$ and a covariance matrix $\mathbf{W}=\operatorname{diag}\left\{\left(\Sigma / \gamma_{\boldsymbol{s}_{1}}^{\text {old }}\right)^{-1}, \cdots,\left(\Sigma / \gamma_{\boldsymbol{s}_{L}}^{\text {old }}\right)^{-1}\right\}$. Then, the optimization problem of (16) is equivalent to minimize the objective function of

$$
\boldsymbol{f}(\boldsymbol{x})=\left\|\boldsymbol{e}(\boldsymbol{x})^{T} \mathbf{W} \boldsymbol{e}(\boldsymbol{x})\right\|
$$

The minimum value of $f(x)$ occurs at the point with the gradient being zero, i.e. $\frac{\partial \boldsymbol{f}(\boldsymbol{x})}{\partial \boldsymbol{x}}=0$. Since the function (17) includes both the independent variable and the parameters, the closed form solution to the gradient is not straightforward to derive. Alternatively, by directly approximating the nonlinear $\boldsymbol{h}_{s}(\boldsymbol{x})$ by a Jacobi matrix, i.e. $\mathbf{H}_{\boldsymbol{s}}(\boldsymbol{x})=\frac{\partial \boldsymbol{h}_{\boldsymbol{s}}(\boldsymbol{x})}{\partial \boldsymbol{x}^{T}}=\frac{\boldsymbol{c}_{\boldsymbol{s}}-\boldsymbol{x}}{\left\|\boldsymbol{c}_{\boldsymbol{s}}-\boldsymbol{x}\right\|}$, an iterative weighted least square method (IWLS) [15] can be implemented to solve the optimization problem. The IWLS method is numerically robust, which can easily make use of almost any kind of measurement for the purpose of positioning in mobile location setting [15]. A disadvantage of the IWLS method is that it requires an initial guess of the position. Poor setting of the initial position may cause the algorithm to converge to a local maximum value of the likelihood, resulting in a location divergent from the real position, which corresponds to the global maximum value of the likelihood.

\section{B. Initial Value Settings}

For the lack of the a-priori information about the position of $\boldsymbol{x}$, the initial estimation of position $\boldsymbol{x}_{0}$ can be taken as the average of all the estimated positions from different permutations, i.e., $\hat{\boldsymbol{x}}_{0}=\mathbf{E}\left(\hat{\boldsymbol{x}}_{\boldsymbol{s}}\right)$, where the expectation is in terms of all the possible permutations of the emitters $s$. Given that there are in total $L$ permutations, we have $x_{0}=\frac{1}{L} \sum_{\boldsymbol{s}} \hat{x}_{\boldsymbol{s}}$.

\section{Weight Threshold}

In order to accelerate the computing, a weight threshold $\gamma_{\text {threshold }}$ can be introduced to decide whether a permutation $\boldsymbol{s}_{j}$ is to be considered for further computing. Specifically, after an E-step, when the weight $\gamma_{\boldsymbol{s}_{j}}^{\text {old }}$, as seen in (14), satisfies $\gamma_{\boldsymbol{s}_{j}}^{\text {old }}<\gamma_{\text {threshold }}$, we may assume that the permutation $\boldsymbol{s}_{j}$ has a very small probability to match the current estimate $x^{\text {old }}$ and the measurements $z$. Therefore, $s_{j}$ can be deleted from the candidate permutation list for further consideration.
TABLE I

TESTING SCENARIOS

\begin{tabular}{c|c|ccc|c}
\hline Emitter & cite & Longitude & Latitude & Altitude & Tx power (W) \\
\hline 1 & Grande Etoile & $43.38368 \mathrm{~N}$ & $5.42607 \mathrm{E}$ & 582 & $10 \mathrm{k}-100 \mathrm{k}$ \\
2 & La Mille & $43.27815 \mathrm{~N}$ & $5.49003 \mathrm{E}$ & 260 & 36 \\
3 & Super Rouviere & $43.25051 \mathrm{~N}$ & $5.57462 \mathrm{E}$ & 206 & 6 \\
4 & Promegues & $43.27443 \mathrm{~N}$ & $5.30804 \mathrm{E}$ & 55 & 3500 \\
5 & Mont Des Marseille & $43.35890 \mathrm{~N}$ & $5.57462 \mathrm{E}$ & 610 & 20 \\
\hline
\end{tabular}

\section{Algorithm Description}

In summary, our EMP algorithm can be stated as follows, which jointly identifies the DVB-T SFN emitters and estimates the position of the receiver.

\section{Algorithm 1 EMP algorithm}

Initialization: the initial mobile positioning $\hat{x}^{0}$ is set based on Section III-B, set the stopping error tolerance threshold to $\delta$, and set the maximum number of iterations to $N_{\max }$; for $k=1, \cdots, N_{\max }$,

1) compute the weights $\gamma_{\boldsymbol{s}_{j}}^{\text {old }}$ of the permutations according to (14);

2) delete the permutations having small weights, according to what stated in Section III-C;

3) update the estimate $\hat{\boldsymbol{x}}_{k}$ according to (16);

4) If $\left\|\hat{\boldsymbol{x}}_{k}-\hat{\boldsymbol{x}}_{k-1}\right\|>\delta$ and $k \leq N_{\max }$, set $k=k+1$ and return Step 2). Otherwise, stop and return the estimate to the mobile position.

\section{end for}

\section{TESTS RESUlTS}

\section{A. Test Scenario}

The scenario used to test the EMP algorithm is selected in the city of Marseille in Southern France. According to the public information released by the Conseil Supérieur de L'audiovisuel (CSA) [16], there are five emitters covering the DVB-T SFN in the area. Table I lists the information of all the five emitters. From Table I, we can know that the emitter of the Grande Etoile is the main transmitter in the area. The signals are transmitted in terms of the Effective Radiated Power (ERP) in the range of $10 \mathrm{KW}$ to $100 \mathrm{KW}$ for different channels. The transmitter in Promegues is the second most powerful one in the area with an ERP of $3.5 \mathrm{KW}$. There are three emitters in the suburban of the city, whose transmission power is relatively small and in the range from $6 \mathrm{~W}$ to $36 \mathrm{~W}$.

In [7], a TOA estimation approach has been proposed, which is capable of tracking weak DVB-T signals for the purpose of ranging, even in the case that the received signals are not strong enough to meet the requirement for the quasi error free $(\mathrm{QEF})$ demodulation. This is because the TOA estimation method in [7] exploits the pilots of DVB-T signals for tracking, which are transmitted with boosted power in comparison to the signals for payload data. As an example, Fig. 2 shows one snapshot of the channel acquisition in one of the field tests provided by the software defined DVB-T receiver developed in [7]. In this test, the receiver is located in a parking place 


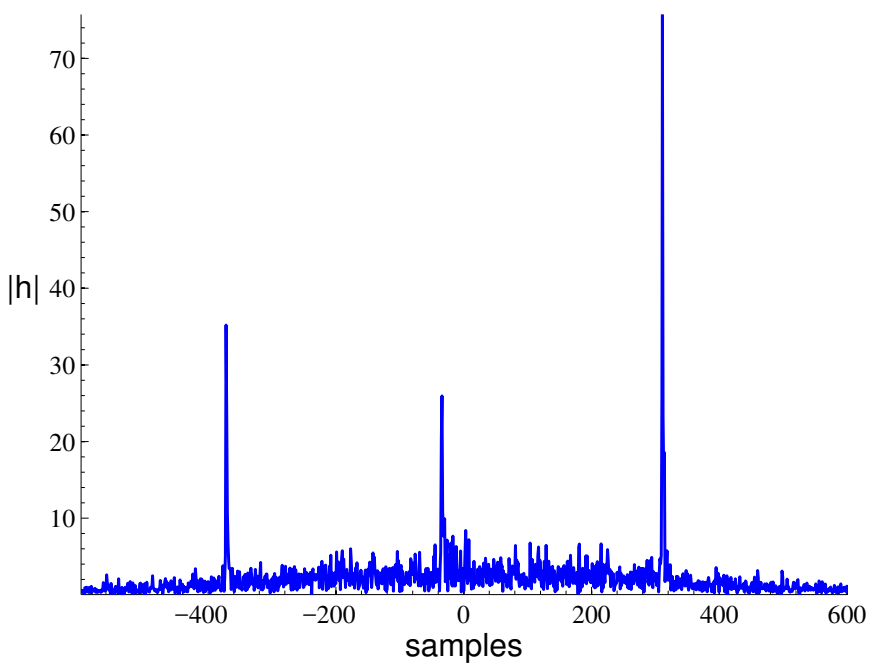

Fig. 2. Snapshot of the channel acquisition from one field test in Marseille, where the receiver is located in a parking place near the Notre Dame de la Garde and the acquired signals are transmitted in the frequency band of Channel 30

near the Notre Dame de la Garde, having the two dimensional (2D) coordinate of [43.28481 N, 5.371708 E] and the altitude of 129 meters, according to a GPS receiver. Additionally, the central carrier frequency of the acquired signal is 546.1667 $\mathrm{MHz}$, which is the central frequency of Channel 30.

\section{B. One Realization of the Test}

To evaluate the performance of our EMP algorithm, we assume that there is a receiver, which is located within the line of sight (LOS) region of the four DVB-T SFN emitters noted in Table I and Fig. 3. The measurement noise is assumed to be the white Gaussian noise distributed with zero mean and a variance of $\sigma_{n}^{2}=10 \mathrm{~m}^{2}$. This assumption is in consistent with the field test results in high signal-noise ratio (SNR) region, as shown in [7]. In Fig. 3, we show a one-step realization of the EMP algorithm, when the initial position is randomly distributed within the considered area. By contrast, in Fig. 4, we depict the 3D surface of the likelihood evaluated from (5). In our evaluation for Fig. 4, all the possible permutations in $\mathbf{S}$ for a specific grid $x_{i}$ are considered. For the lack of the knowledge, equal a-priori probability of $p\left(\boldsymbol{s}=\boldsymbol{s}_{j}\right)=1 / L$ is assumed to compute the likelihood.

From Fig. 3 and Fig. 4, it is observed that in high SNR region, positioning is very reliable with small evaluation error, meaning that all the emitters can be correctly identified. Comparatively, when the SNR decreases, some of the emitters might not be correctly identified, as shown in Fig. 5, where the noise variance is $100 \mathrm{~m}^{2}$. However, as shown in Fig. 5, the position of the receiver estimated by the EMP algorithm still converges to the actual position of the receiver, even though more iterations are required, and when some of the emitters are not correctly identified by the EMP algorithm.

\section{Statistical Performance}

Fig. 6 depicts the root mean square errors (RMSE) performance of the EMP algorithm, when different numbers of

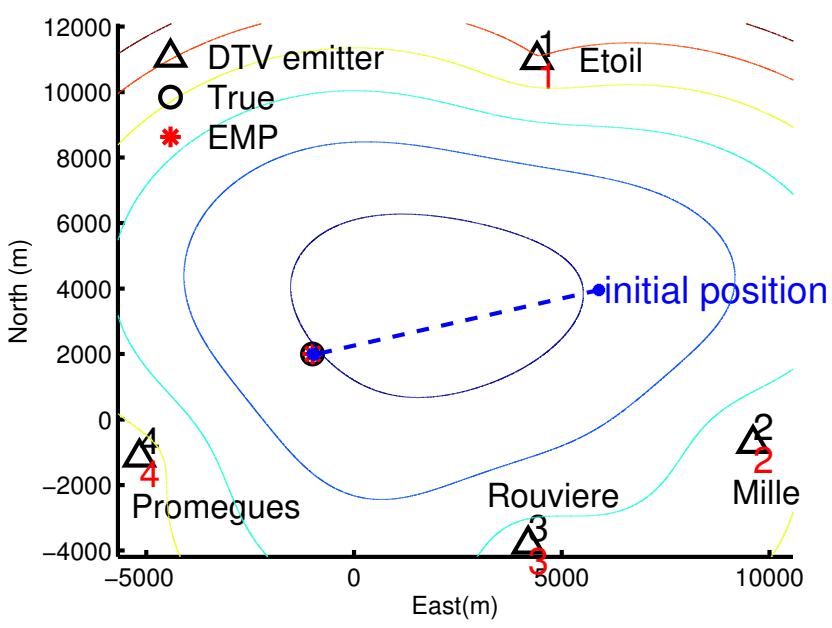

Fig. 3. One realization of the EMP algorithm experiencing small measurement noise, where the variance of measurement noise is $10 \mathrm{~m}^{2}$. In the test, the positioning error is within $2.5 \mathrm{~m}$ and the positioning error is computed as the distance between the user's true position and the position estimated by the EMP algorithm. There are two numbers marked with each emitter. The upper (black) number is the true label of the emitter, while the lower (red) number is the label of the emitter estimated by the EMP algorithm. In this test, all the labels of the emitters are correctly identified.

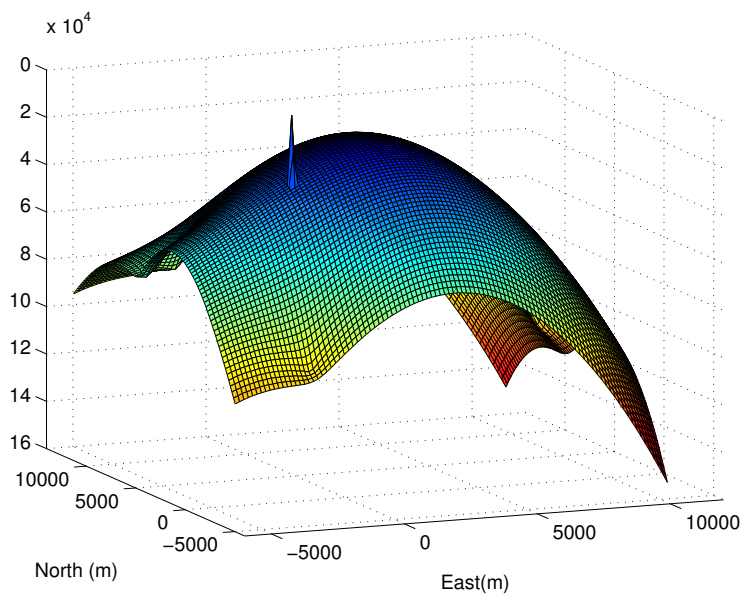

Fig. 4. 3D surface of the values related to the likelihood evaluated by (5). In order to have a good view of the global minimum in the 3D surface, the coordinate of $z$ axis increases from the top to the bottom.

emitters are deployed. The results were obtained from the average of 2000 Monte Carlo realizations. In our simulations, the position of the receiver is randomly generated within the area of interest. In order to compare the performance of the EMP algorithm, the Cramér-Rao lower bound (CRLB) [17], [18] was computed and also shown in Fig. 6. We should note that, the CRLB was computed under the ideal knowledge that the receiver knows which signal is received from which emitter. Therefore, the CRLB is an over-optimistic estimation for the problem considered in this contribution.

From the results of Fig. 6, we observed that when only 3 emitters are used for positioning, the positioning error is much higher than the CRLB. By contrast, when there are four or five emitters available for positioning, the positioning performance 


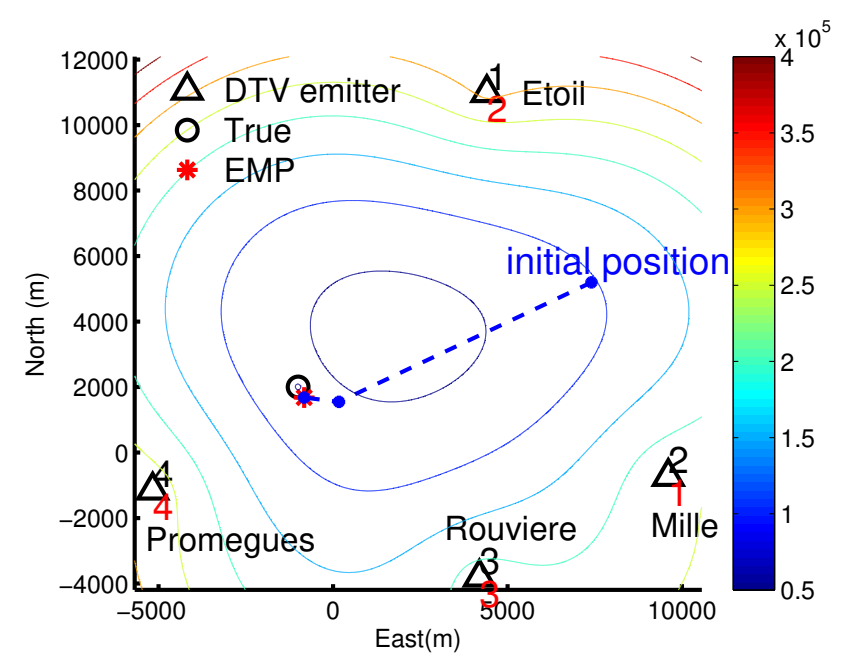

Fig. 5. One realization of the EM assisted positioning under large measurement noise, where the variance of measurement noise is $100 \mathrm{~m}^{2}$. In this test, the positioning error is $10.1 \mathrm{~m}$ and the two emitters of Etoil and Mille are not correctly identified.

is close to the CRLB.

As shown in Fig. 6, when the number of emitters increases, the performance of positioning improves. In contrast to the significant performance improvement when the number of emitters is increased from three to four, there is only marginal improvement, when the number of emitters is increased from four to five. Therefore, our proposed EMP algorithm is a highly effective positioning algorithm even when the receiver experiences the emitter confusion problem. Furthermore, considering the complexity-performance trade-off, in practice, it is desirable for the EMP algorithm to work with four emitters, as evidenced by the results shown in Fig. 6 .

In Fig. 7, we show the error rate of the emitter identification, when three, four or five emitters are employed by the EMP algorithm. Explicitly, when the test uses 3 emitters, the error rate of the emitter identification is the largest among the three test scenarios. This is because, in this case, there are not enough pseudo-ranges provided for the EMP algorithm. By contrast, when four or five emitters are used in the tests, the error rate of emitter identification decreases significantly. However, it is interesting to find that the error rate of emitter identification in the case of 5 emitters is slightly larger than that in the case of 4 emitter. In fact, this is because, when more emitters are used in the tests, the emitter confusion problem becomes severer, resulting in the increased error rate of emitter identification. Nevertheless, as shown in Fig. 6, the slightly larger error rate of the emitter identification has little effect on the final positioning accuracy, which is inferred by the EMP algorithm. This is due to the fact that the objective function of the EMP algorithm is to minimize the positioning error, while the labels of the emitters are the hidden variables to be identified. Therefore, from the results of Fig. 6 and Fig. 7, we are implied that the EMP algorithm is robustness to the errors of emitter identification. Furthermore, the results of Fig. 6 and Fig. 7 suggest that it is highly efficient for the EMP algorithm to work with four emitters, especially when we also take the

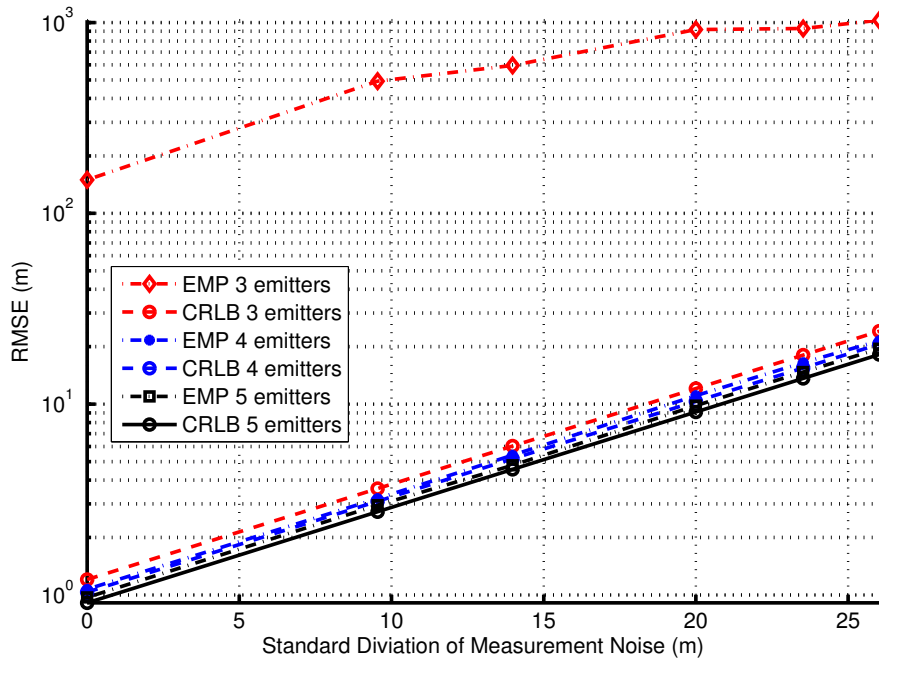

Fig. 6. Positioning performance of the EMP algorithm experiencing Gaussian distribution measurement noise

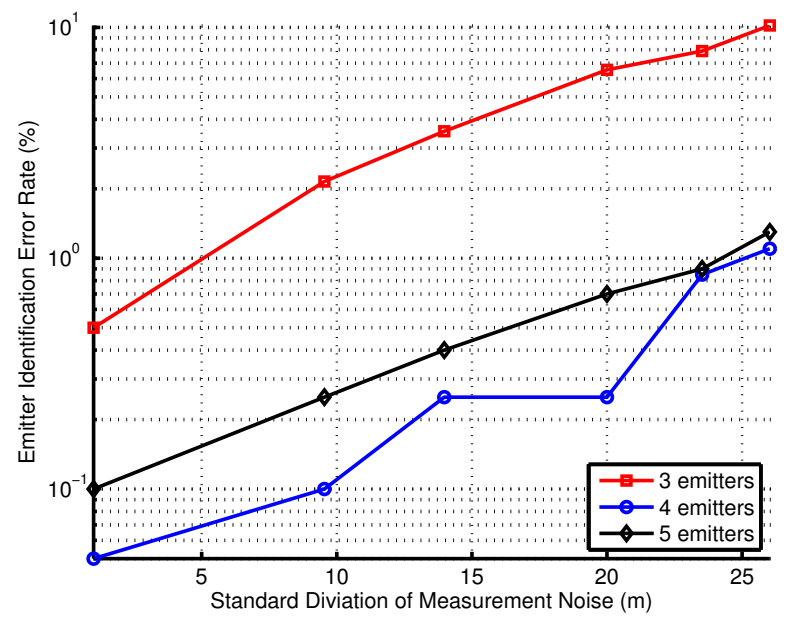

Fig. 7. Error rate of emitter identification.

implementation complexity into account.

\section{CONCLUSIONS}

This paper has studied the wireless positioning in DTV networks. Due to the SFN property in DTV networks, the receiver cannot identify the specific emitter where a received signal comes. Therefore, an EMP algorithm has been proposed to jointly estimate the receiver position and identify the labels of the emitters. To evaluate the algorithm, a test scenario in the city of Marseille has been considered, where the positions of five emitters are set according to the official information published by the Authority in France. The performance of the EMP algorithm has been studied by considering different issues. Our studies and simulation results show that, in the case of 4 or 5 emitters, the EMP algorithm is highly effective to achieve good positioning performance, even in the existence of the emitter confusion problem. When 4 or 5 emitters are available, the statistical performance shows that the EMP algorithm is capable of achieving the performance approaching 
the CRLB that is only achievable with full emitter information. Furthermore, with 4 or 5 emitters, the EMP algorithm is also effective to identify the DTV emitters. Additionally, our simulation tests show that the EMP algorithm is robust to the tolerance for the errors of emitter identification. Note that, the methodology proposed in this work is general, which may be applied in the other SFNs for TOA based positioning.

\section{ACKNOWLEDGMENT}

This work was partially supported by Academy of Finland under the Grant No. 254232. Liang Chen would like to thank SIGNAV Laboratory in École Nationale de L'Aviation Civile (ENAC), France, for providing the assistance on the field test in Southern France.

\section{REFERENCES}

[1] E. D. Kaplan, Ed., Understanding GPS: Principles and Applications. Norwood: Artech House, 1996.

[2] M. Rabinowitz and J. Spilker, "A new positioning system using television synchronization signals," IEEE Transactions on Broadcasting, vol. 51, no. 1, pp. 51 - 61, March 2005.

[3] Framing structure, channel coding and modulation for digital terrestrial television, ETSI Standard Std. ETSI EN 302744 V1.1.4., January 2001.

[4] J. T. Ong, H. Yan, S. V. Rao, and G. Shanmugam, "Indoor DTV reception: measurement techniques," IEEE Transactions on Broadcasting, vol. 50, pp. 192-199, 2004.

[5] M. Rabinowitz and J. Spilker, "Positioning using the ATSC digital television signal," U.S.A. Patent WO: 2004/057360 A2, 2004-08-07.

[6] L. Chen, L.-L. Yang, and R. Chen, "Time delay tracking for positioning in DTV networks," in Proc. Ubiquitous Positioning, Indoor Navigation, and Location Based Service (UPINLBS), 2012, Oct. 2012, pp. 1-4.

[7] L. Chen, O. Julien, P. Thevenon, D. Serant, A. G. Pe?na, and H. Kuusniemi, "TOA estimation for positioning with DVB-T signals in outdoor static tests," IEEE Tranactions on Broadcasting, vol. 61, no. 4, pp. 625638, December 2015.

[8] L. Chen, P. Thevenon, G. Seco-Granados, O. Julien, and H. Kuusniemi, "Analysis on the TOA tracking with DVB-T signals for positioning," IEEE Tranactions on Broadcasting, vol. 62, no. 4, pp. 957 - 961, December 2016.

[9] X. Wang, Y. Wu, and J. Y. Chouinard, "A new position location system using DTV transmitter identification watermark signals," EURASIP Journal on Applied Signal Processing, vol. 2006, pp. 1-11, 2006.

[10] J. Yang, X. Wang, M. Rahman, S. I. Park, H.-M. Kim, and Y. Wu, “A new positioning system using DVB-T2 transmitter signature waveforms in single frequency networks," IEEE Transactions on Broadcasting, vol. 58, no. 3, pp. 347-359, 2012.

[11] P. Thevenon, "S band air interfaces for navigation system: a focus on OFDM signals," Ph.D. dissertation, Universite de Toulouse, 2010.

[12] J. Yan and L. Wu, "Base station identification in single-frequency network positioning system under mixed line-of-sight/non-line-of-sight condition," IET Communications, vol. 6, no. 18, pp. 3189-3195, December 2012.

[13] A. B. Gelman, J. S. Carlin, H. S. Stern, and D. B. Rubin, Bayesian Data Analysis, 2nd ed. Chapman \& Hall, 2000.

[14] A. Dempster, N. Laird, and D. Rubin, "Maximum likelihood from incomplete data via the EM algorithm," Journal of the Royal Statistical Society, Series B, vol. 39, no. 1, p. 1?38, 1977.

[15] N. Sirola, "Closed-form algorithms in mobile positioning: Myths and misconceptions," in Proceedings of 7th Workshop on Positioning Navigation and Communication (WPNC), 2010, Bremen, Germany, Oct. 2010, pp. 38-44.

[16] "tvignaud.pagesperso-orange.fr/tv/tnt.pdf."

[17] S. M. Kay, Fundamentals of Statistical Signal Processing: Estimation Theory. Prentice Hall, 1993.

[18] K. W. Cheung, H. C. Sho, W. K. Ma, and Y. T. Chan, "Least squares algorithms for time-of-arrival-based mobile location," IEEE Transactions on Signal Processing, vol. 52, no. 4, pp. 1121-1128, April 2004. 